\title{
Nachruf auf Prof. Dr. med. Dr. h.c. Walter Siegenthaler
}

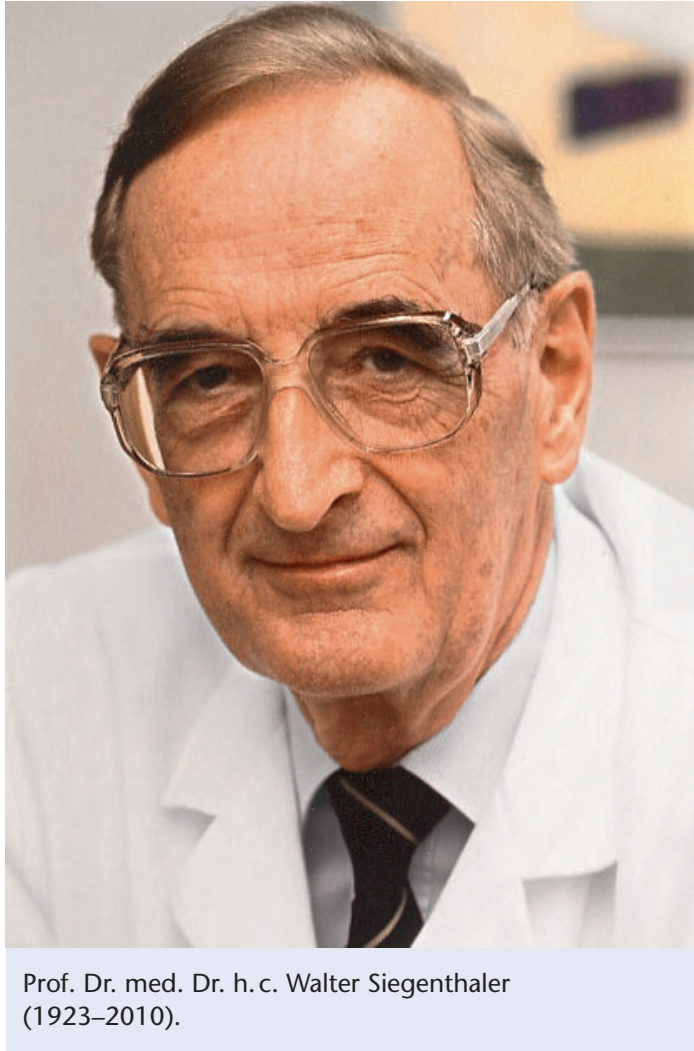

Am 24. Oktober 2010 ist Prof. Dr. med. Dr. h.c. Walter Siegenthaler, emeritierter Ordinarius für Innere Medizin an der Universität Zürich, kurz vor Vollendung seines 87 . Lebensjahres verstorben.

Walter Siegenthaler wurde 1923 in Davos geboren; dort durchlief er die Primarschule und anschliessend in Chur das Gymnasium. Von 1942 bis 1948 studierte er in Zürich Medizin. Nach dem Staatsexamen leistete er die militärischen Kaderschulen - für ihn eine selbstverständliche Pflicht, die er bis zum Divisionsarzt der Zürcher Feld-Division 6 im Grade eines Oberstleutnants erfüllt hat. Anschliessend trat er seine erste Assistentenstelle an der Zürcher Pathologie unter den Professoren von Meyenburg und Zollinger an. Es folgte die Medizinische Klinik des damaligen Kantonsspitals Zürich unter Prof. Dr. W. Löffler. 1954 ging Walter Siegenthaler als Oberarzt mit Prof. Dr. Robert Hegglin nach St. Gallen. Dieser war soeben zum Chefarzt der Medizinischen Klinik des dortigen Kantonsspitals gewählt worden. Vier Jahre später, 1958, wurde Hegglin zum Ordinarius für Innere
Medizin an die Universität Zürich und zum Chefarzt der Medizinischen Poliklinik berufen. Walter Siegenthaler folgte ihm zusammen mit seiner Frau, Dr. med. Gertrud Siegenthaler, die in St. Gallen als Assistenzärztin tätig war und die er 1957 geheiratet hatte.

1961 habilitierte sich Walter Siegenthaler für das Fach der Inneren Medizin und trat dann zusammen mit seiner Frau einen Forschungsaufenthalt an der Stanford University School of Medicine in Palo Alto und anschliessend am Cornell University Medical College in New York City an. 1963 kehrten Siegenthalers nach Zürich zurück.

Als Stellvertreter von Prof. Robert Hegglin baute Walter Siegenthaler ein Labor auf, das sich mit dem neuentdeckten Renin-Angiotensin-System befasste. 1967 erfolgte die Beförderung zum Extraordinarius.

1968 wurde Walter Siegenthaler zum Ordinarius und Direktor der Medizinischen Poliklinik der Universität Bonn berufen. Kurze Zeit danach starb Robert Hegglin in Zürich unerwartet, und Walter Siegenthaler erhielt 1970 den Ruf, dessen Nachfolge in Zürich anzutreten, gleichzeitig aber auch als Ordinarius und Direktor an die Medizinische Klinik in Bonn zu wechseln. Der Entscheid fiel ihm schwer, und Bonn versuchte alles, um ihn zu halten. Schliesslich entschloss er sich, der Berufung nach Zürich zu folgen.

In Zürich baute Walter Siegenthaler die Medizinische Poliklinik zu einer international renommierten Institution aus. Dabei wurde er von seiner Frau Gertrud und einem grossen Mitarbeiterstab unterstützt. Es ist ihm dabei gelungen, die Einheit des Faches Innere Medizin zu bewahren und gleichzeitig die sich allmählich entwickelnden Subspezialitäten unter der Leitung von Leitenden Ärzten und Extraordinarien zu fördern. Manche dieser Abteilungen haben wissenschaftliche Pionierleistungen vollbracht. Es sei hier bloss das Beispiel von Dr. Andreas Grüntzig erwähnt, der 1977 in Zürich die weltweit erste Ballondilatation eines Herzkranzgefässes durchgeführt hat.

Von 1978 bis 1980 war Prof. W. Siegenthaler Dekan der Medizinischen Fakultät und von 1980 bis zu seiner Emeritierung 1991 Vorsitzender der Klinikdirektorenkonferenz. 
Bei all seinen zahllosen nationalen und internationalen Verpflichtungen in Gesundheits-, Hochschulund Forschungspolitik hatten für Walter Siegenthaler zwei Bereiche immer absolute Priorität, nämlich die Betreuung seiner Patienten und der Unterricht für seine Studenten. Die Zeit der Vorlesungen während des Semesters war ein Tabu, das kaum je einer Sitzung oder einem Kongress zum Opfer fiel. Walter Siegenthaler hat seine Vorlesungen sorgfältig vorbereitet und sie mit dem logischen Aufbau des Stoffes und dem Miteinbezug der Studenten zu einem besonderen Erlebnis gemacht. Dieselbe strikte Disziplin galt für den täglichen 11-Uhr-Rapport im Ambulatorium und die wöchentliche Chefvisite auf der Bettenstation. Schwierige und unklare Fälle waren immer ein Grund, ihn in einer Besprechung oder Sitzung zu stören, und er liess es sich nie nehmen, sofort ans Krankenbett zu kommen. Die Chefvisiten waren eindrückliche didaktische Feuerwerke, bei denen die Assistenten und Studenten nicht nur in der Differentialdiagnostik trainiert wurden, sondern der Chef auch korrekte «Bedside manners» durchsetzte, die obligatorische Krawatte für die Herren eingeschlossen! Er war ein Patron alter Schule, und zartbesaitete Assistenzärzte durchliefen eine harte Lehre.

Eine ganz besondere Gabe Walter Siegenthalers war die Didaktik. Er verstand es meisterhaft, komplexe pathophysiologische Zusammenhänge klar und verständlich darzustellen. An Kongressen und Fortbildungskursen gelang es ihm, das in unserem Lande doch oft recht gehemmte Publikum zur Diskussion anzuregen. Er hat den Votanten stets das Gefühl vermittelt, ihre Fragen seien von besonderer Bedeutung, und hat, wenn nötig, hochtrabende Podiumsteilnehmer vom hohen Ross heruntergeholt.

Ein Höhepunkt der Klinikwoche in Zürich waren die jeden Freitagabend stattfindenden interdisziplinären Fallbesprechungen, das legendäre «Sigirama», bei dem Patienten mit komplexen Krankheitsbildern vorgestellt und von den Vertretern der verschiedenen Fächer diskutiert wurden. Hier dirigierte Walter Siegenthaler virtuos das Orchester der vielen medizinischen Solisten und Primadonnen im Bestreben, eine für den Patienten heilsame Symphonie zu schaffen.

Die wohl berühmtesten Produkte von Walter Siegenthalers didaktischer Begabung sind seine Lehrbücher, die im deutschsprachigen Raum in keinem Assistentenbüro und in kaum einer Arztpraxis fehlen; es seien hier nur die «Differentialdiagnose Innerer Krankheiten» und die «Klinische Pathophysiologie» erwähnt. Beide Werke erlebten zahlreiche Auflagen und waren die eigentlichen Hausmarken der Zürcher Medizinischen Poliklinik. Seine «Differentialdiagnose» wurde mittlerweile auch auf Chinesisch und Russisch übersetzt.

Dass ein Mann vom Format Walter Siegenthalers mit Ehrungen überhäuft wird, ist selbstverständlich. So wurde er 1991 zum Ehrendoktor der Universität Halle ernannt. Eine ganz besondere Auszeichnung war 1983 seine Wahl zum Präsidenten der Deutschen Gesellschaft für Innere Medizin. Diese Ehre wurde seit der Gründung der Gesellschaft bloss zwei Schweizern zuteil. In dieselbe höchste Kategorie gehört auch die Ernennung zum Ehrenmitglied der Association of American Physicians im Jahre 2003.

Im Jahre 2003 hat Walter Siegenthaler die «Walter und Gertrud Siegenthaler Stiftung» gegründet, die junge Wissenschaftlerinnen und Wissenschaftler der Medizin in ihrer Karriere unterstützt. Die Förderung des medizinischen Nachwuchses war ihm stets ein grosses Anliegen.

Mit Walter Siegenthaler hat uns einer der letzten grossen Schweizer Internisten verlassen. Sein Erbe lebt aber in seinen Schülern, seiner Stiftung und in seinen Büchern weiter.

Prof. Dr. Rolf A. Streuli, Langenthal 